\title{
Dell Hymes
}

\section{The Scope of Sociolinguistics}

https://doi.org/10.1515/ijsl-2020-2084

Abstract: As sociolinguistics continued to develop in the 1970s, members of the Council's Committee on Sociolinguistics (1963-1979) reflected on the direction and intellectual impact of this emergent discipline. In this 1972 article, Dell Hymes, cochairman of the committee, describes several orientations toward the field among its practitioners, and argues for what he regarded as the most ambitious: a "socially constituted linguistics." By this, Hymes meant a sociolinguistics that challenges linguistics' core theoretical starting points of linguistic structure and grammar with a focus on the social meaning and functions of language in context. In relation to our "Sociolinguistic Frontiers" series, Hymes presciently argues that ultimately the field must address how inequality and language intersect, going "beyond means of speech and types of speech community to a concern with persons and social structure."

The term "sociolinguistics" began to gain currency about ten years ago. The subsequent decade has seen a great deal of activity. There have been general symposia; symposia on major topics; notable major research efforts; the launching of series of working papers; books of readings, increasingly specific to the field; textbooks; even a series of collected papers of middle-aged men who find themselves senior scholars; and journals.

The present meeting is in a way a culmination of the decade's activity. Where do we stand? How far have we progressed? In some ways, very far. In one fundamental regard, I think, simply to a threshold.

We are all familiar with the gap that can exist between public concerns and the competence of scientists. The energetic activity in sociolinguistics is nourished in important part by the obvious relevance of much of its subject matter, joining other academic fields in which concern for education, children, ethnic relations, and governmental policies find expression. However, there are scientific as well as practical needs. If relevance to social problems were not recognized, sociolinguistic research would still be needed for the sake of an adequate theory of language. Some of what is done under the rubric of sociolinguistics may be justified only in the sense that something is better than nothing, when need is great. But in the present state of

Dell Hymes, University of Pennsylvania, USA 
sociolinguistics, I would maintain (1) that the scientific as well as the practical side of linguistics stands in need; (2) that scientific and practical needs converge; and (3) that steps taken during the past decade have brought us to the threshold of an integrated approach to linguistic description. As to (1), witness the current disarray with regard to arguments in syntax and semantics and to the place of semantics, intonation, and even phonology and lexicon in a model of grammar itself, as issues of empirical adequacy and validity are pressed against the dominant "intuitionist" approach-and as other, contextually oriented traditions of work are gradually reinvented or grudgingly rediscovered. As to (2), note that findings about the organization of variation and the structure of speech acts-both are central to linguistic theory-contribute to the scientific basis that successful practice needs. At the same time, facts of practical experience (e. g., the organization of linguistic features in terms of verbal repertoires; the role of social meaning as a determinant of acceptability and the "creative aspect of language use"; the effects of personal identity, role, and setting as constraints on competence) point to severe limitations of present linguistic theory and stimulate efforts to overcome them. As to (3), if we take "integrated" to encompass the structure of sentences within the structure of discourse, of referential meaning within the meanings of speech acts, and of dialects and languages within the organization of verbal repertoires and speech communities, then we can see a convergence implicit in much of the best recent work and envisage a unity it can attain.

\section{Orientations and concerns in sociolinguistics}

The term sociolinguistics means many things to many people, and of course no one has a patent on its definition. Indeed, not everyone whose work is called sociolinguistic is ready to accept the label, and those who use the term include and emphasize different things. Nevertheless, three main orientations can be distinguished, orientations that can be labeled: the social as well as the linguistic; socially realistic linguistics; socially constituted linguistics. Let me characterize each of these in relation to linguistic theory.

The social as well as the linguistic. Here may be placed ventures into social problems involving language and the use of language, which are not seen as involving a challenge to existing linguistics. American linguistics does have a tradition of practical concerns-one can mention Sapir's semantic research for an international auxiliary language, Bloomfield's work in the teaching of reading, Swadesh's literacy work, the "Army method" of teaching foreign languages. The salient examples today involve American cities and developing nations and 
concern problems of education, minority groups, and language policies. For the most part this work is conceived as application, lacking theoretical content, or else as pursuing theoretical concerns that are in addition to those of normal linguistics, or perhaps even wholly unrelated to them. When sociolinguistics serves as a legitimizing label for such activity, it is, as said, not conceived as a challenge to normal linguistics; linguists who perceive such a challenge in the label tend to eschew it.

Socially realistic linguistics. ${ }^{1}$ This term is apt for work that extends and challenges existing linguistics with data from the speech community. The challenge, and indeed the accomplishment, might be summed up in the two words, variation and validity. An outstanding example is the work of William Labov, whose orientation toward linguistics is represented in his recently published papers. The expressed theoretical concerns are not distinct from those of normal linguistics, e. g., the nature of linguistic rules and of sound change, but the method of work and the findings differ sharply. Here might also be classified work in which dependence of the analysis of meaning and speech acts on social context is recognized.

Socially constituted linguistics. This orientation is less developed than the first two but represents, I think, the fundamental challenge to which sociolinguists have come. The phrase "socially constituted" is intended to express the view that social function gives form to the ways in which linguistic features are encountered in actual life. With this assumption, an adequate approach must begin by identifying social functions and discover the ways in which linguistic features are selected and grouped together to serve them. Such a point of view cannot leave normal linguistic theory unchallenged (as does the first orientation), nor limit its challenge to reform, because its own goals are not allowed for by normal theory and cannot be achieved by "working within the system." A socially constituted linguistics shares the practical concerns of other orientations; it shares concern for social realism and validity; but even if it could wait for the perfection of a "linguistic theory" of the normal sort, it could not then use such a theory. Many of the features and relationships with which a socially constituted linguistics must deal would never have been taken up in that kind of theory. (That is why, indeed, "linguistic theory" of the normal sort is not a "theory of language," but only a theory of grammar.) A socially constituted linguistics is concerned with social as well as referential meaning, and with language as part of communicative conduct and social action. Its task is the thoroughgoing critique of received notions and practices, from the standpoint of

1 I owe this term to Maxine Bernstein, in whose dissertation in progress (at the University of Pennsylvania) I encountered it. 
social meaning, that is, from a functional perspective. Such a conception reverses the structuralist tendency of most of the twentieth century, toward the isolation of referential structure, and the posing of questions about social functions from that standpoint. The goals of social relevance and social realism can be fully accomplished only from the standpoint of the new conception, for much of what must be taken into account, much of what is there-organized and used-in actual speech can only be seen, let alone understood, when one starts from function and looks for the structure that serves it.

I have given examples to support this thesis in earlier papers. Here let me merely mention the following instances:

From a comprehensive functional standpoint, a phonetic feature such as aspiration appears to be a true phonological universal, specialized to referential function in some languages, and to stylistic function in others (hence not of indifference to general theory in its role in English).

Recognition of a social-identifying function motivates an independently controllable articulation otherwise left unintelligible.

The status of a sentence as a speech act depends upon the rights and obligations, roles and statuses, of the participants.

Unless one extends the rules governing a verbal summons in English to include nonverbal acts (a knock, a telephone ring), a significant generalization is lost; similarly, the function of deixis in San Blas Cuna is served by a set of forms that includes lip pointing.

Speech probably serves to mark sex-role status in every community, but linguists hitherto have discovered it only when intrusive in a normal grammatical description.

Some consistent ways of speaking make use of the resources of more than one language (e.g., the Dutch of Surinam blacks, who impose a norm that is grammatically and lexically standard, but phonologically creole).

In some communities, distinct languages can be described as lexically distinct with a common grammar and phonology (Kupwar approaches this).

The semantic structure represented by a choice of pronoun in one community may be expressed by a choice of dialect in another, and choice of language in still a third, so that analysis of the function from a universal standpoint cannot stay with one part of language, or even within the category language.

In sum, if our concern is social relevance and social realism, we must recognize that there is more to the relationship between sound and meaning than is dreamt of in normal linguistic theory. In sound there are stylistic as well as referential features and contrasts; in meaning there is social as well as referential import; in between there are relationships not given in ordinary grammar but there for the finding in social life. 
It is not that phenomena pointing to a more general conception of the relationship between sound and meaning have not long been noted, and often enough studied with insight and care. Expressive language, speech levels, social dialects, registers, functional varieties, code- and style-switching are familiar and essential concepts; and the interlocked subjects of stylistics, poetics, and rhetoric have flourished in recent years. Anything that can be accomplished in theory and method for a socially constituted linguistics must incorporate and build on that work, which has done much to shape what I write here. But the tendency has been to treat such phenomena and such studies as marginal or as supplementary to grammar. The hegemony of grammar as a genre and that of the referential function as its organizing basis have been preserved. The essence of a functional approach is to take function as problematic, not for granted; to assume as part of a universal theory of language that a plurality of functions are served by linguistic features in any act and community; to require validation of the relationships between features and functions, and of their organization into varieties, registers, ways of speaking, ethnographically within the community; and to take functional questions-a functional perspective-as having priority, that is, as being fundamental, both in general theory and in specific accounts, to whatever can be validly said as to structure, competence, universals, etc.

Such a perspective was present in the structuralism of the period before World War II and has never been wholly lost. In Anglo-American circles it has begun to come to the fore in work under the aegis of sociolinguistics in recent years. Salient examples include the work of Labov on "sociolinguistic structure," of John Gumperz on verbal repertoire, of Basil Bernstein on codes, of Joshua Fishman on domains, of Norman Denison and R. B. Le Page on multilingualism, and of Susan Ervin-Tripp on sociolinguistic rules. What is important here is the element in each work that contributes to a general methodological perspective. Such work goes beyond the recognition and analysis of particular cases to suggest a mode of organization of linguistic features other than that of a grammar. The common implication which I want to emphasize and elaborate is, in its weaker form, that such alternative modes of organization exist; and, in its stronger form, that one or more such alternative modes of organization may be fundamental.

\section{The speech community}

There is a second point, linked to the first, and owing its full recognition to much the same body of work: a conception of the speech community not in terms 
of language alone (especially not just one language, and a fortiori not just one homogeneous language).

Many linguists, although they would find the wording odd, might accept a definition of the object of linguistic description as the organization of features within a community. From the present standpoint the wording is not odd, but vital. The two points just stated in negative terms can now be put positively:

(1) The organization of linguistic features within a speech community is in terms of ways of speaking within a verbal repertoire.

(2) Membership in a speech community consists in sharing one (or more) ways of speaking.

The often stated foundation of linguistic theory, that in a speech community some utterances are the same, differing only in "free" variation, and that the goal of theory is to explain what counts as contrast and what does not, has perhaps served the development of linguistics well in its purely "referential" interpretation. One bird of function in the hand, so to speak, may have been preferable to entering the bush to cope with two. But, to elaborate the figure, it appears that neither bird will fly without the other, even that neither is itself a whole bird. To pursue the figure no doubt too far, the bird in the hand proves to be a featherless monopteron, to be restored only out of the ashes of conventional grammar. The true foundation of theory and method is that in a speech community some ways of speaking are the same, that some of the persons talk the same way.

A community, then, is to be characterized in terms of a repertoire of ways of speaking. Ways of speaking are to be characterized in terms of a relationship between styles, on the one hand, and contexts of discourse, on the other. The formal concept underlying speech styles is what Ervin-Tripp has called rules of co-occurrence. The formal concept of relating speech styles to contexts of discourse is called by her rules of alternation. The speech styles defined by rules of co-occurrence draw on the linguistic varieties present in a community, from whose resources they select and group features in sometimes complex ways. The relationships dubbed rules of alternation are in the first instance considerations of appropriateness, and of marked and unmarked usage.

The recognition of Ervin-Tripp of speech styles themselves as the elements of a further system of rules is comparable in nature and importance to the earlier recognition of grammatical transformations (as rules operating on rules). The study of the structure of relationships among speech styles opens up the possibility of a generative approach; and it makes the study of social meaning as embodied in roles, activities, and situations integral to the explanation of the meanings of the speech styles themselves. 
Linguistics of course does not itself command analysis of social role, activities, and situations. Of this, two things can be said. First, such analysis is necessary. There really is no way that linguistic theory can become a theory of language without encompassing social meaning, and that signifies becoming a part of the general study of communicative conduct and social action. Second, this step is dictated by the development of linguistics itself. Having begun its structural course at the far side of meaning, with a focus on phonology, linguistics has proceeded through successive foci on morphology, syntax, semantics, and now performative and speech acts. There is no way to analyze speech acts adequately without ethnography; no language is a perfect metalanguage for the acts that can be performed with it. The study of speech acts can indeed be a center of a socially constituted linguistics, but its own logic broaches the general study of the vocabulary of action, in communities and in social science. Again, if we take seriously Chomsky's implicit call for linguistics to concern itself with the "creative aspect" of language use, and with the basis of the ability to generate novel yet appropriate sentences, we again are forced into analysis of setting as well as syntax. For appropriateness is not a property of sentences, but of a relationship between sentences and contexts, especially with regard to the property of "creativity"-whether that is saying something new in a familiar setting or something familiar in a setting that is new. At every turn, it almost would seem, linguistics is wrestling with phenomena and concepts that turn out to entail relationships, only one pole of which is within linguistics' usual domain. The true generalizations can never be captured except from a perspective that encompasses both poles.

To bring out this point one may say that a socially constituted linguistics has as a goal a kind of explanatory adequacy complementary to that proposed by Chomsky. Chomsky's type of explanatory adequacy leads away from speech, and from languages, to relationships possibly universal to all languages and possibly inherent in human nature. It is an exciting and worthwhile prospect. The complementary type of adequacy leads away from what is common to all human beings and all languages toward what particular communities and persons have made of their means of speech. It is comparative and evolutionary in a sociocultural, rather than biological, sense. It sees as in need of explanation the differential elaboration of means of speech, and of speech itself. At a surface level it notices gross contrasts in speech activity, from great volubility to great taciturnity; gross contrasts in elaboration of message form; gross contrasts in the predominance of traditional and of spontaneously encoded utterance; gross contrasts in the complication, or simplification, of the obligatory surface structure of languages themselves. These contrasts, and the typologies to which they point, no doubt find their explanation at a deeper level. Rules of conduct in 
relation to roles and settings; the role of a language variety in socialization or in boundary maintenance; values, conceptions of the self, and beliefs as to the rights and duties one owes to others as fellow members of a community-all will be found to have a place. The general problem, then, is to identify the means of speech and ways of speaking of communities; to find, indeed, where are the real communities, for language boundaries do not give them, and a person or a group may belong to more than one; to characterize communities in terms of their repertoires of these; and through ethnography, comparative ethnology, historical and evolutionary considerations, to explain something of the origin, development, maintenance, obsolescence, and loss of ways of speaking and types of speech community-of the face speech wears for human beings before they learn that it is language, a thing apart, and the property of linguists.

This complementary goal of explanatory adequacy comes not, it must be admitted, from the internal logic of linguistics, but from an external aspiration. Chomsky's type of explanatory adequacy, to be sure, would seem to owe much to his own concern to understand the human mind and to revitalize rationalist philosophy. He has made his concern an effective goal for many in linguistics, philosophy, and psychology. The concern that motivates explanation directed toward ways of speaking and speech communities may or may not find a similar response. This concern, put simply, is with human liberation.

If linguistic research is to help as it could in transcending the many inequalities in language and competence in the world today, it must be able to analyze inequalities. In particular, a practical linguistics so motivated would have to go beyond means of speech and types of speech community to a concern with persons and social structure. If competence is to mean anything useful (we do not really need a synonym for grammar), it must refer to the abilities actually possessed by persons. A salient fact about a speech community, realistically viewed, is the unequal distribution of abilities, on the one hand, and of opportunities for their use, on the other. This appears to be an old story in mankind, e. g., a cursory look at the globe discloses that definition of women as communicatively second-class citizens is widespread. When, where and what they may speak, the conceptions of themselves as speakers with which they are socialized, show again and again that from the community point of view they at least are not "ideal speakers," though they may on occasion be ideal hearers.

The goal of explanatory adequacy with regard to speech communities as comprising ways of speaking will be quite enough, I suppose, for most linguists to consider, let alone to accept. Yet, I believe, if linguistics is to realize its potential for the well-being of mankind, it must go even further and consider speech communities as comprising not only rules, but also sometimes 
oppression, sometimes freedom, in the relation between personal abilities and their occasions of use.

\section{Conclusion}

What, then, is the scope of sociolinguistics? Not all I have just described but, rather, that part of it which linguists and social scientists leave unattended. The final "goal" of sociolinguistics, I think, must be to preside over its own liquidation. The flourishing of a hybrid term such as sociolinguistics reflects a gap in the disposition of established disciplines with respect to reality. Sometimes new disciplines do grow from such a state of affairs, but the recent history of the study of language has seen the disciplines adjacent to a gap grow themselves to encompass it. Some can recall a generation ago when proper American linguists did not study meaning, and ethnographers had little linguistic method. A study of meaning in another language or culture (say, grammatical categories or kinship terms) could qualify as "ethnolinguistic" then. Today of course semantics is pursued in both linguistics and ethnography, and a mediating interdisciplinary label is unnecessary; "semantics" itself will usually suffice.

Let us hope for a similar history for sociolinguistics. In one sense the issue again is the study of meaning, only now, social meaning.

In 1934, Sapir wrote: “The social psychology into which the conventional cultural and psychological disciplines must eventually be resolved is related to these paradigmatic studies as an investigation into living speech is related to grammar. I think few cultural disciplines are as exact, as rigorously configurated, as self-contained as grammar, but if it is desired to have grammar contribute a significant share to our understanding of human behavior, its definitions, meanings, and classifications must be capable of a significant restatement in terms of a social psychology which...boldly essays to bring every cultural pattern back to the living context from which it has been abstracted in the first place...back to its social matrix."2

Sapir had begun to rethink the nature of language, culture, and society from a standpoint he sometimes called "psychiatric," or "social psychology," and which today we might more readily label the standpoint of social interaction, or communicative conduct: the standpoint, as I would see it, of sociolinguistics. Obviously, Sapir's intellectual lead did not prevail after his death in 1939,

2 Edward Sapir, "The Emergence of the Concept of Personality in a Study of Cultures," Journal of Social Psychology 5, no. 3 (1934): 408-410. 
although its influence can be traced in many quarters. Such a fact must humble expectation. But a decade ago I did venture to predict:

"It may be that the development of these foci of interest [semantic description, sociolinguistic variation] will lead historians of twentieth-century linguistics to say that whereas the first half of the century was distinguished by a drive for the autonomy of language as an object of study and a focus upon description of structure, the second half was distinguished by a concern for the integration of language in sociocultural context and a focus upon the analysis of function."3

Item Editor's note: The author is Professor of Folklore and Linguistics at the University of Pennsylvania, a member of the board of directors of the Social Science Research Council, and the chairman of its Committee on Sociolinguistics, which, with support provided by the National Science Foundation, cosponsored with Georgetown University its 23rd Annual Round Table on Languages and Linguistics, March 16-18, 1972. This article is a condensed version of the paper Dell Hymes presented at the meeting. The full version will be published in Roger W. Shuy, ed., Sociolinguistics: Current Trends and Prospects (Georgetown University Monograph Series on Languages and Linguistics, No. 25, 1972), by Georgetown University Press, with whose permission this version is printed here. The other members of the Committee on Sociolinguistics are Charles A. Ferguson, Stanford University; Allen D. Grimshaw, Indiana University; John J. Gumperz, University of California, Berkeley; William D. Labov, University of Pennsylvania; staff, David Jenness. The idea of a conference on the state of the field of sociolinguistics was conceived by Charles Ferguson, chairman of the committee 1963-70.

Article note: This archival document has been reproduced the way it was initially published, with no further editing nor formatting of the bibliography.

3 Dell Hymes, ed., Language in Culture and Society (New York: Harper \& Row, 1964), 11. 